\title{
DEVELOPING AND TESTING OPERATIONAL DEFINITIONS FOR FUNCTIONAL AND HIGHER ORDER DRIVING INSTRUCTION
}

\author{
Johnathon Ehsani ${ }^{1}$, Bruce G. Simons-Morton ${ }^{1} \&$ Sheila G. Klauer ${ }^{2}$ \\ ${ }^{1}$ Eunice Kennedy National Institute of Child Health and Human Development \\ Bethesda, MD, USA \\ ${ }^{2}$ Virginia Tech Transportation Institute \\ Blacksburg, VA, USA \\ johnathon.ehsani@nih.gov, mortonb@exchange.nih.gov\& CKlauer@vtti.vt.edu
}

Summary: The amount and type of driving instruction provided to novice teen drivers during the learner period may be associated with future crash risk. The purpose of this study was to (1) operationally define two types of driving instruction: functional and higher order instruction, and to (2) test these definitions in a sample of newly licensed novice teenage drivers during the first ten hours of supervised driving. Functional driving instruction was defined as instruction that relates to the present time or immediate future; and related to specific events that are occurring during the drive itself. Higher order driving instruction was instruction that could be extrapolated to a future driving situation; that conveys general principles of driving related to potential events that occur. These operational definitions were tested in conversation occurring during driving instruction in a sample of 90 teen drivers, recruited within three weeks of receiving their learner permit. Teen drivers' vehicles were equipped with microphones; conversations were recorded and coded for each type of instruction that was observed. As expected, parents provided substantial driving-related instruction on a variety of topics. During the first ten hours of supervised driving only $17.5 \%$ of observed driving-related instructions was higher order. This test provides face validity of the operational definitions of driving instruction. These definitions may assist in quantifying the type and amount of driving instruction occurring during the supervised practice stage of licensure, and provide an empirical basis for evaluating the association between driving instruction and independent driving performance.

\section{INTRODUCTION}

Motor vehicle crashes are the leading cause of death and a leading cause of injury for teens in the United States (Centers for Disease Control and Prevention 2014). However, teenage drivers' crash risk is not uniformly high during the early stages of driving. While novices are receiving direct driving supervision during the learner permit phase, their crash risk is very low (Williams 2003). In contrast, crash risk during the first months of independent driving is very high (McCartt, Shabanova et al. 2003). One hypothesis that has been proposed for this pronounced change in crash risk is that supervisors (typically parents) may not be providing instruction that would equip novice teen drivers with the necessary skills for safe independent driving.

In a number of survey studies, teenage drivers described their parents' instruction as being focused on imparting vehicle handling skills (Tronsmoen 2011, Mirman and Kay 2012). A recent 
naturalistic study of parental driving instruction described two different types of instruction occurring during supervision (Goodwin, Foss et al. 2014): (1) "functional instruction related to vehicle handling or operation" or (2) "instruction related to a higher order understanding of driving". In that study, parents were observed providing focus instruction on functional activities such as vehicle handling, rather than on higher order tasks such as identifying potentially hazardous situations or managing a safety gap between vehicles. The amount of instruction related to vehicle handling/operation decreased over time, suggesting teens were developing the sufficient skills to require less guidance for these basic maneuvers.

This seminal evaluation of instruction based on video-based naturalistic methods provided the first objective information about how parents instruct their adolescent children as they learn to drive. However, Goodwin and colleagues did not provide operational definitions of functional and higher order driving instruction that could be used in different driving scenarios. The purpose of this study was (1) to develop operational definitions of functional and higher order driving instruction, and (2) test these definitions in a sample of naturalistic driving that occurred during the first ten hours of supervised driving among newly licensed novice teenage drivers.

\section{METHODS}

A panel of researchers with expertise in teenage driving developed the operational definitions of functional and higher order driving instruction. Their task also involved developing examples of instruction under different driving conditions. These definitions were pilot tested by applying them as rules to a coding protocol for naturalistic driving video reduction.

The definitions were applied to the first ten hours of driving under supervision in a sample of newly licensed drivers. The primary vehicles of 90 teen drivers (41 males and 49 females, mean age 15.6 years) were instrumented with data acquisition capabilities within three weeks of obtaining a learner's permit. A microphone recorded all conversations in the vehicle and four cameras recorded video images. Participants were instructed to drive as they would normally.

Sample Recruitment: The study required the participation of teenage drivers and at least one of their parents. Recruitment was conducted in local newspaper and high schools in southwestern Virginia, USA. Participants were initially screened in a telephone interview for eligibility using the following inclusion criteria: (a) being between 15.5 and 16.1 years old; (b) holding a learner permit driver's license allowing supervised driving for no more than two weeks; (c) having at least one parent willing and able to participate; (d) access to a vehicle expected to survive mechanically for at least 18 months; (e) residing within a one hour drive of the research center or satellite location; and (f) holding liability insurance on the vehicle to be used in the study (required by state law).

Participants were excluded during the prescreen telephone interview if they: (a) had a diagnosis of attention deficit disorder (ADD) or attention deficit hyperactivity disorder (ADHD); (b) had an identical twins (which would make it difficult to distinguish participants during coding); and (c) needed to enter restricted areas (i.e., that do not allow cameras for security reasons). 
Coding Protocol: To track the progression of driving instruction over time, a 30 second driving segment was randomly selected from each consecutive five-minute period of driving during the first ten hours of practice. Each participant generated 12 driving clips per hour, totaling 120 driving clips per participant for the first ten hours of practice driving. The segments were recorded when the ignition was on, and could include conversations while parked provided the ignition remained on. For each recorded segment, the conversation between parents and teens was coded for each type of driving instruction. When a trip duration was not divisible by 5 minutes, then the last minutes of the trip would not be sampled (e.g. if a trip was 24 minutes long, the last 4 minutes would not be sampled). If audio was not clear or audible to the coders, the segment would be excluded from the sample.

Five trained coders viewed each clip and categorized the topics and nature of driving instruction. Coders were trained using a documented coding protocol with the coding manager reviewing $100 \%$ of their work until the trainee coder was achieving over $95 \%$ accuracy. Following the initial training, the data coding manager would spot check the coders and conduct intra-rater testing of observed segments.

Coders were instructed to listen to the conversation and make a determination based on the content of the dialogue as to whether it was directed by the supervisor or the teen driver. If the randomly selected video clip began in the middle of the conversation, the coder was instructed to review the 30 seconds of sampled audio and provide an assessment based upon that 30 seconds of time. The reductionist could review the audio prior to the 30 seconds to gain context but needed to base the responses to the coding exclusively on the 30 -second segment.

Coders were provided with the descriptions of instruction to classify the 'topic' of driving instruction. An inter-rater test indicated $86 \%$ average accuracy for all coders as compared to an expert coder. The inter-rater test consisted of each rater coding 30 epochs on 80 variable collected in the protocol.

\section{RESULTS}

Extending the definition provided by Goodwin and colleagues, functional driving instruction was defined as instruction that is related to the present time or immediate future related to specific events that are occurring during the drive itself. This instruction is dependent on the driving situation. Higher order driving instruction was instruction that could be extrapolated to a future driving situation; that may not be applied directly but conveys general principles of driving related to potential events that may occur. This type of instruction may result from a specific event, but generalizes to other situations.

These definitions were testing on randomly selected driving segments, from participants' first ten hours of practice driving. A total of 120 segments were recorded for each of the ninety participants, totaling 10,800 observations. In approximately $7 \%$ of clips, audio was absent or data were missing. In the final sample of 10,049 segments, an adult supervisor (typically a parent) was present in the vehicle in $97.2 \%$ of instances. In segments where a supervisor was present $(n=9,767)$, conversation occurred in $87.3 \%$ of clips, and the supervisor typically spoke more frequently that the teenage driver $(68.5 \%)$. 
Based on the observed conversation between supervisors and teens, driving instruction was classified according to the operational definitions of functional or higher order instruction. In some instances, both types of instruction were provided consecutively. In these instances, both types of instruction were recorded as occurring, resulting in an additional category: Combination of functional and higher order instruction. Table 1 lists the topics of instruction that supervisors provided during the first ten hours of driving, and classifies the instruction according to functional $(82.5 \%)$, higher order $(10.5 \%)$, or a combination of both $(7.0 \%)$.

Functional instruction was highest when supervisors were navigating and guiding teens on vehicle handling. The highest percentage of higher order instruction was observed when supervisors were remarking on vehicle behavior, discussing vehicle handling, and giving warnings or pointing out hazards. Examples of the operational definitions, according to topic, and the type of instruction are provided in Table 2.

Table 1: Topics and Type of Driving Instruction during the First 10 Hours of Practice Driving

\begin{tabular}{|c|c|c|c|c|c|c|}
\hline \multirow[b]{3}{*}{ Vehicle Handling or Operation } & \multirow{2}{*}{\multicolumn{2}{|c|}{$\begin{array}{c}\text { Functional } \\
\text { Instruction }\end{array}$}} & \multirow{2}{*}{\multicolumn{2}{|c|}{$\begin{array}{c}\begin{array}{c}\text { Higher Order } \\
\text { Instruction }\end{array} \\
\mathrm{N}(\text { row } \%)\end{array}$}} & \multirow{2}{*}{$\frac{\text { Combination }^{\wedge}}{\mathrm{N}(\text { row } \%)}$} & \multirow{3}{*}{$\begin{array}{c}\text { Total } \\
\mathrm{N} \\
3145\end{array}$} \\
\hline & & & & & & \\
\hline & 2673 & $(84.9)$ & 210 & $(6.7)$ & $262 \quad(8.3)$ & \\
\hline Remarking on Driving Behavior & 1292 & $(74.3)$ & 298 & $(17.2)$ & $148 \quad(8.5)$ & 1738 \\
\hline Navigation & 1614 & $(94.1)$ & 68 & $(4.0)$ & $33 \quad(1.9)$ & 1715 \\
\hline Give Warning/Detect Hazard & 971 & $(75.2)$ & 201 & $(15.6)$ & $119 \quad(9.2)$ & 1291 \\
\hline Rules of the Road & 691 & $(78.1)$ & 134 & $(15.1)$ & $60 \quad(6.8)$ & 885 \\
\hline Asks Question About Driving Task & & $(80.0)$ & 17 & $(18.9)$ & $1 \quad(1.1)$ & 90 \\
\hline Total & 7313 & $(82.5)$ & 928 & $0.5)$ & $623(7.0)$ & 8864 \\
\hline
\end{tabular}


Table 2: Topic of instruction and examples of functional and higher order driving instruction

\begin{tabular}{|c|c|c|}
\hline Topics & $\begin{array}{l}\text { Example of functional driving } \\
\text { instruction }\end{array}$ & $\begin{array}{l}\text { Examples of higher order } \\
\text { driving instruction }\end{array}$ \\
\hline $\begin{array}{l}\text { Navigation (giving/receiving } \\
\text { turn-by-turn directions) }\end{array}$ & $\begin{array}{l}\text { You're going to make a left turn } \\
\text { at the next traffic light }\end{array}$ & $\begin{array}{l}\text { I like to think about the route } \\
\text { I'm going to take before I leave } \\
\text { home; before you start driving } \\
\text { think about where you are going } \\
\text { and how to get there; I avoid } \\
\text { that route because of the traffic }\end{array}$ \\
\hline $\begin{array}{l}\text { Give Warning/Detect Hazard } \\
\text { (warning of upcoming hazards } \\
\text { or driving hazards in general) }\end{array}$ & $\begin{array}{l}\text { You've got a car pulling out of a } \\
\text { drive way up ahead. }\end{array}$ & $\begin{array}{l}\text { You've got to adjust to the road } \\
\text { situation. If it rains, you've got } \\
\text { to adjust to that. } \\
\text { When you drive in the } \\
\text { neighborhood, watch for tail } \\
\text { lights indicating a vehicle may } \\
\text { pull out of a drive way. } \\
\text { Always check your blind spot. }\end{array}$ \\
\hline $\begin{array}{l}\text { Vehicle Handling or Operation } \\
\text { (mechanics of turning, braking, } \\
\text { speed selection, lane keeping, } \\
\text { merging, lane changing, } \\
\text { following behavior) }\end{array}$ & $\begin{array}{l}\text { Slow down. Slow way down. } \\
\text { He's pulling out. } \\
\text { Put on your turn signal and when } \\
\text { safe move into the far lane. } \\
\text { Go now. }\end{array}$ & $\begin{array}{l}\text { You tend to follow too closely. } \\
\text { Try leaving a few car spaces } \\
\text { between yourself and the other } \\
\text { vehicle while you drive. }\end{array}$ \\
\hline $\begin{array}{l}\text { Remarks about driving } \\
\text { behavior }\end{array}$ & $\begin{array}{l}\text { It's ok, you didn't hit the curb. } \\
\text { You took that corner too fast. }\end{array}$ & $\begin{array}{l}\text { When turning, be sure you are } \\
\text { well over in the far lane so no } \\
\text { vehicle could mistakenly pull } \\
\text { around you. } \\
\text { It is best to signal at least } 1 / 2 \\
\text { block before you turn or merge. }\end{array}$ \\
\hline $\begin{array}{l}\text { Asks question about driving } \\
\text { task }\end{array}$ & $\begin{array}{l}\text { What are you going to do you } \\
\text { when that bus stops ahead of } \\
\text { you? } \\
\text { Did you see that car? }\end{array}$ & $\begin{array}{l}\text { How often should you be } \\
\text { checking your rear view mirror? }\end{array}$ \\
\hline $\begin{array}{l}\text { Rules of the road (stating or } \\
\text { quizzing/asking teen about } \\
\text { rules) }\end{array}$ & $\begin{array}{l}\text { That's not a median, it's a yellow } \\
\text { line. } \\
\text { What does that sign mean? } \\
\text { Stop for that school bus and don't } \\
\text { pass. }\end{array}$ & $\begin{array}{l}\text { What do you do when you see } \\
\text { lights flashing amber? } \\
\text { How can you determine that a } \\
\text { green light may be about to turn } \\
\text { amber? } \\
\text { What should you do if a school } \\
\text { bus is stopped ahead of us? }\end{array}$ \\
\hline
\end{tabular}




\section{DISCUSSION}

The purpose of this paper was twofold. The first was to develop operational definitions for functional and higher order driving instruction. The second was to test these definitions in a sample of naturalistic driving among novice teenage drivers receiving supervision. We found that driving instruction was typically focused on an immediate or functional driving task, with particular emphasis on vehicle handling and navigation, rather than on developing higher order instruction. Although previous research had established that much of supervised driving instruction does not involve higher-order instruction (Goodwin, Foss et al. 2014), this paper provides clear evidence and examples of the differences in functional and higher-order instruction

Previous research has established that the first few hours of practice driving correspond to the period when basic vehicle control skills can be taught to novice drivers (Hall 1996). Our finding that the first ten hours of driving instruction were focused on the functional aspects of the driving task provides face validity of these operational definitions of instruction, and aligns with Fitts and Posner's early stages of skill development (Fitts and Posner 1967). In this model, the learner gains an basic understanding about the nature of driving, experiences initial trials using this information, and begins to refine driving skills.

The appropriate amount of functional or higher order instruction is likely to change as a learner gains experience, develops skills and encounters increasingly complex driving situations. The earliest driving instruction given to a novice driver is more likely to be about vehicle handing and control-level operations, while higher-order instruction would be more beneficial after the novice has gained some experience and mastery of vehicle control. Therefore, our finding that $17.5 \%$ of driving instruction was dedicated to higher order concepts may be appropriate to the learning stage of the drivers in sample.

Despite many states in the U.S. requiring a number of supervised practice hours (Insurance Institute for Highway Safety 2014), relatively little is understood about the role of driving instruction in teen driver safety. The appropriate amount of instruction, and the proportion of functional versus higher order instruction needed to reduce novice teen driver crashes are also unknown. While there is likely to be considerable variability among teens in the amount and type of instruction required to provide a safety benefit, higher order instruction is likely to benefit novice teen drivers by providing components of a mental schema that teens can utilize while driving (Piaget 1971).

Providing higher order instruction requires the conscious articulation of automatic behaviors. Parents themselves may not be aware of their own cognitive processing of driving situations, and therefore have difficulty imparting that information to the driver they are supervising. If an association between the amount and type of instruction and novice teen drivers' crash risk is established, the development of tools for parents to provide higher order instruction may be warranted. 


\section{CONCLUSION}

The operational definitions of two types of driving instruction: functional and higher order, were developed and assessed. As expected, parents provided substantial driving-related instruction on a variety of topics, though during the first ten hours of supervised driving, only $17.5 \%$ of observed driving-related instructions were higher order. It is hoped these provisional definitions may establish a basis to consistently categorize driving instruction, and may stimulate refinement of the categories and topics. These definitions may assist in quantifying the type and amount of driving instruction occurring during the supervised practice phase of licensure, and in establishing an association between driving instruction and crash risk.

\section{ACKNOWLEDGEMENT}

This research was supported by the Intramural Research Program of the NICHD, contract \# N01HD-5-3405.

\section{REFERENCES}

Centers for Disease Control and Prevention. (2014). "WISQARS Injury Mortality Reports, 1999 - 2012." Retrieved 2nd December, 2014, from http://www.cdc.gov/injury/wisqars/fatal_injury_reports.html.

Fitts, P. M. and M. I. Posner (1967). Human performance. Belmont, CA, Brooks/Cole Publishing Company.

Goodwin, A. H., R. D. Foss, L. H. Margolis and S. Harrell (2014). "Parent comments and instruction during the first four months of supervised driving: An opportunity missed?" Accident Analysis \& Prevention 69(0): 15-22.

Hall, J. (1996). "Role of formal instruction and informal practice in learning to drive." Ergonomics 39(4): 693-706.

Insurance Institute for Highway Safety. (2014). "Graduated Driver Licensing." Retrieved 30th July, 2014, from http://www.iihs.org/iihs/topics/laws/graduatedlicenseintro?topicName=teenagers.

McCartt, A. T., V. I. Shabanova and W. A. Leaf (2003). "Driving experience, crashes and traffic citations of teenage beginning drivers." Accident Analysis and Prevention 35(3): 311-320.

Mirman, J. H. and J. Kay (2012). "From Passengers to Drivers: Parent Perceptions About How Adolescents Learn to Drive." Journal of Adolescent Research 27(3): 401-424.

Piaget, J. (1971). Biology and Knowledge. Edinburgh., Edinburgh University Press: 6-7.

Tronsmoen, T. (2011). "Differences between formal and informal practical driver training as experienced by the learners themselves." Transportation Research Part F: Traffic Psychology and Behaviour 14(3): 176-188.

Williams, A. F. (2003). "Teenage drivers: patterns of risk." Journal of Safety Research 34: 5-15. 